Alexander Beremiz Hilario Tacuri

\title{
Desempenho de sistemas OFDM em canais não-lineares variantes no tempo
}

Tese apresentada ao Programa de Pós-graduação em Engenharia Elétrica do Departamento de Engenharia Elétrica da PUC-Rio como requisito parcial para obtenção do título de Doutor em Engenharia Elétrica.

Orientador: Prof. José Mauro Pedro Fortes 


\section{Alexander Beremiz Hilario Tacuri}

\section{Desempenho de sistemas OFDM em canais não-lineares variantes no tempo}

Tese apresentada ao Programa de Pós-graduação em Engenharia Elétrica do Departamento de Engenharia Elétrica do Centro Técnico Científico da PUC-Rio como requisito parcial para obtenção Do título de Doutor em Engenharia Elétrica. Aprovada pela Comissão Examinadora abaixo assinada.

Prof. José Mauro Pedro Fortes

Orientador

Centro de Estudios em Telecomunicações / PUC-Rio

Prof. Raimundo Sampaio Neto

Centro de Estudos em Telecomunicações / PUC-Rio

Prof. Marco Antonio Grivet Mattoso Maia

Centro de Estudos em Telecomunicações / PUC-Rio

Prof. Pedro Henrique Gouvêa Coelho

UERJ

Prof. Ernesto Leite Pinto

IME

Prof. Paulo Roberto Rosa Lopes Nunes

IME

Prof. José Eugenio Leal

Coordenador Setorial do Centro Técnico Científico - PUC-Rio 
Todos os direitos reservados. É proibida a reprodução total ou parcial do trabalho sem autorização da universidade, do autor e do orientador.

\section{Alexander Beremiz Hilario Tacuri}

Graduou-se em Engenharia Eletrônica (Universidad Nacional de San Agustin) em 2008. Recebeu o título de Mestre em Engenharia Elétrica na area de Sistemas de Comunicação pela PUC-Rio em 2010.

Ficha Catalográfica

Hilario Tacuri, Alexander Beremiz

Desempenho de sistemas OFDM em canais não-lineares variantes no tempo/ Alexander Beremiz Hilario Tacuri; orientador: José Mauro Pedro Fortes. - 2014.

104 f: il. (color.) ; $30 \mathrm{~cm}$

Tese (doutorado) - Pontifícia Universidade Católica do Rio de Janeiro, Departamento de Engenharia Elétrica, 2014.

Inclui bibliografia.

1. Engenharia Elétrica - Teses. 2. OFDM. 3. Nãolinearidade com memória. 4. Canal multipercurso. I. Fortes, José Mauro Pedro. II. Pontifícia Universidade Católica do Rio de Janeiro. Departamento de Engenharia Elétrica. III. Título. 
Aos alicerces da minha vida: Meu Pai, minha Mãe e à minha Irmã. 


\section{Agradecimentos}

Sería imposible intentar escribir, en tan poco espacio, la importancia que mis padres: Donato e Guillermina, tuvieron en mi formación humana y académica. Su amor, constante incentivo y demostración de la importancia y frutos de los estudios y del arduo trabajo, no solo en palabras, sino principalmente en ejemplos, fueron fundamentales en el largo proceso educacional que me permite concluir esta ultima etapa de mis estudios. A ellos, les debo todo.

Também, gostaria de expressar minha profunda gratidão ao meu orientador Professor José Mauro Pedro Fortes por seu apoio, orientação e as muitas horas que ele passou editando e corrigindo meu trabalho. Suas palavras de incentivo me mantiveram motivado ao longo destes anos e aprendi muito com ele tanto profissional como pessoalmente. Essa conquista não teria sido possível sem a ajuda dele.

Também serei eternamente grato à minha irmã, Patricia, não só por me apoiar na conclusão desta Tese, mas pela oportunidade que me deu para iniciá-la. Muito obrigado, irmã.

Também gostaria de agradecer aos meus colegas e amigos do CETUC que fizeram minha permanência no Brasil maravilhosa.

Finalmente, gostaria de agradecer o apoio financeiro provido pela Coordenação de Aperfeiçoamento de Pessoal de Nível Superior - CAPES. 


\section{Resumo}

Hilario Tacuri, Alexander Beremiz; Fortes, José Mauro Pedro. Desempenho de sistemas OFDM em canais não-lineares variantes no tempo. Rio de Janeiro, 2014. 104p. Tese de Doutorado

- Departamento de Engenharia Elétrica, Pontifícia Universidade Católica do Rio de Janeiro.

O desempenho de sistemas de comunicações modernos que utilizam técnicas de modulação multiportadora como, por exemplo, o OFDM (Orthogonal frequency division multiplexing), é altamente sensível às distorções nãolineares geradas principalmente por amplificadores de alta potência (HPAs). De modo a caracterizar completamente estas distorções, os efeitos de memória destes dispositivos têm que ser considerados. Estes efeitos tornam-se mais importantes à medida que a largura de banda do sinal aumenta. Por outro lado, o aumento na duração do símbolo dos sistemas multiportadora torna-o mais sensível às variações de tempo dos canais móveis. Em particular o efeito doppler tende a prejudicar a ortogonalidade entre as sub-portadoras, gerando interferências entre sub-portadoras (Inter-Carrier Interference - ICI). Assim, torna-se importante o estudo dos efeitos de não-linearidades e canais variantes no tempo em sistemas OFDM, principalmente em sistemas de comunicações móveis. Os trabalhos atualmente disponíveis na literatura se limitam a avaliar os efeitos de não linearidades invariantes no tempo ou o efeito de canais lineares variantes no tempo. Assim, no presente trabalho são desenvolvidas expressões matemáticas que permitem avaliar o efeito conjunto de não-linearidades e canais variantes no tempo sobre sinais OFDM. A investigação deste efeito é realizada em dois níveis: efeito sobre o espectro do sinal OFDM e efeito sobre o desempenho do sistema. As expressões analíticas obtidas para ambos os níveis de investigação consideraram a modelagem do canal não-linear variante no tempo por uma Série de Volterra. Resultados numéricos que avaliam o espalhamento espectral e o impacto sobre a taxa de erro de bits em situações específicas são apresentados.

\section{Palavras-chave}

Sistemas OFDM; Comunicações móveis; Não-linearidade; Canal multipercurso. 


\section{Abstract}

Hilario Tacuri, Alexander Beremiz; Fortes, José Mauro Pedro (Advisor). Performance of OFDM systems over timevarying non-linear channels . Rio de Janeiro, 2014. 104p. PhD. Thesis - Departamento de Engenharia Elétrica, Pontifícia Universidade Católica do Rio de Janeiro.

The performance of modern communications systems that use multicarrier modulation techniques such as, for example, OFDM (Orthogonal frequency division multiplexing), is highly sensitive to nonlinear distortions generated mainly by high power amplifiers (HPAs). In order to fully characterize these distortions, the memory effects of these devices must be considered. These effects become more important as the signal bandwidth increases. On the other hand, the increase in symbol duration makes multicarriers systems more sensitive to time variations as those in mobile channels. In particular, the Doppler effect tends to impair the orthogonality between sub-carriers, resulting in inter-carrier interference (ICI). Therefore, the study of the effects of nonlinearities and time-varying channels on OFDM systems performance becomes important, mainly in mobile communications systems. The studies currently available in the literature are limited to evaluating the effects of time invariant non-linearities or the effects of linear time varying channels. In this work, mathematical expressions are developed to evaluate the joint effect of nonlinearities and time-varying channels on OFDM systems. The investigation of these effects is performed at two levels: the effect on the spectrum of OFDM signal and effect on the system's performance. In deriving the analytical expressions related to both levels of investigation the time-varying non-linear channel was modeled by a Volterra Series. Numerical results, obtained for specific situations, illustrate the resulting impact on the OFDM spectrum (spreading) and on the system's bit error rate performance.

\section{Keywords}

OFDM systems; Mobile communications; Non-linearities; Multipath channels. 


\section{Sumário}

1 Introdução $\quad \mathbf{1 2}$

1.1 Breve Histórico OFDM 12

$\begin{array}{ll}1.2 \text { Vantagens do OFDM } & 13\end{array}$

1.3 Problema motivador e escopo da tese 14

$\begin{array}{lll}1.4 & \text { Organização do texto } & 15\end{array}$

2 Visão geral OFDM $\quad 17$

2.1 Princípios do OFDM 17

2.2 Caracterização do sinal OFDM 22

3 Caracterização de sistemas não-lineares com memória 31

3.1 Caracterização de sistemas não-lineares com memória invariantes no tempo 32

3.2 Caracterização de sistemas não-lineares com memória variantes no tempo 36

4 Efeito de não-linearidade variante no tempo sobre o espectro de sistemas OFDM

4.1 Representação do sinal de saída 40

4.2 Caracterização do sinal de saída 42

4.3 Resultados numéricos 46

4.4 Resumo 52

5 Efeito de não-linearidades variante no tempo sobre a probabilidade de erro de sistemas OFDM $\quad 54$

5.1 Receptor OFDM $\quad 55$

5.2 Representação do sinal de entrada ao receptor OFDM 56

5.3 Probabilidade de erro de sistemas OFDM móveis 58

5.4 Caracterização estatística conjunta de $Z_{k, n}$ e $F_{n}$

5.5 Resultados numéricos 69

$\begin{array}{lll}5.6 & \text { Resumo } & 72\end{array}$

$\begin{array}{llr}6 & \text { Conclusões } & 76\end{array}$

$\begin{array}{ll}\text { Referências Bibliográficas } & 78\end{array}$

A Expressões matemáticas relativas à potência do sinal de saída do canal não-linear variante no tempo

A.1 Cálculo da densidade espectral de potência da saída do canal não-linear variante no tempo 85

A.2 Cálculo da potência de saturação de saída da não-linearidade 89

A.3 Relação da Energia do símbolo $\left(E_{s}\right)$ com o Back-Off de saída (OBO) 91

A.4 Relação da Energia do símbolo $\left(E_{s}\right)$ com o Back-Off de entrada (IBO) 93

B Expressões úteis referentes aos kernels variantes no tempo $\quad 94$ 
$\begin{array}{ll}\text { C Caracterização de } N_{k, n} & 96\end{array}$

D Símbolo complexo recebido no intervalo de tempo k e na sub-portadora $\mathrm{n}: R_{k, n}$

E Prova que as variáveis aleatórias $f_{n}\left(\gamma_{i}\right)$ são complexas e conjuntamente gaussianas

F Caracterização de $f_{n}\left(\gamma_{i}\right)$ 


\section{Lista de figuras}

2.1 Estrutura do sistema OFDM baseado em osciladores 18

2.2 Estrutura do sistema OFDM baseado em FTT 20

2.3 Prefixo cíclico do sinal OFDM 21

2.4 DEP da envoltória complexa do sinal OFDM/BPSK com $N=48$, $T=4 \mu s, T_{C P}=0.8 \mu s$ e pulso formatador retangular

3.1 Modelo de Wiener para não-linearidades com memória 33

3.2 Modelo de Hammerstein para não-linearidades com memória 34

3.3 Modelo de Wiener-Hammerstein para não-linearidades com memória 35

3.4 Modelo de Wiener paralelo para não-linearidades com memória 36

3.5 Modelo de não-linearidade com memória variante no tempo 37

4.1 Densidades espectrais de potência com OBO $=3 \mathrm{~dB}$ (IBO $=$ $2.7 \mathrm{~dB}$ ) das envoltórias complexas: (i) sinal de entrada; (ii) sinal de saída (não-linearidade, canal de propagação $\left(f_{d} T / N=0.2\right)$ e filtro de recepção) e; (iii) sinal de saída (não-linearidade e filtro de recepção).

4.2 Densidades espectrais de potência com OBO $=5 \mathrm{~dB}$ (IBO $=$ 8.2dB) das envoltórias complexas: (i) sinal de entrada; (ii) sinal de saída (não-linearidade, canal de propagação $\left(f_{d} T / N=0.2\right)$ e filtro de recepção) e; (iii) sinal de saída (não-linearidade e filtro de recepção).

4.3 Densidade espectral de potência da envoltória complexa do sinal de saída, para diferentes valores de $f_{d} T / N$ e OBO $=3 \mathrm{~dB}(\mathrm{IBO}=2.7 \mathrm{~dB}) 50$

4.4 Densidade espectral de potência da envoltória complexa do sinal de saída, para diferentes valores de $f_{d} T / N$ e OBO $=5 \mathrm{~dB}(\mathrm{IBO}=8.2 \mathrm{~dB}) 51$

4.5 Percentual de potência do sinal de saída do canal considerado, com $\mathrm{OBO}=3 \mathrm{~dB}(\mathrm{IBO}=2.7 \mathrm{~dB})$, fora da faixa de frequências correspondente a uma banda $b$ em torno de $f_{0}$.

4.6 Percentual de potência do sinal de saída do canal considerado, com $\mathrm{OBO}=5 \mathrm{~dB}(\mathrm{IBO}=8.2 \mathrm{~dB})$, fora da faixa de frequências correspondente a uma banda $b$ em torno de $f_{0}$.

5.1 Modelo usado para analisar o impacto da não-linearidade no desempenho de sistemas OFDM móveis

5.2 Regiões de decisão ótimas do BPSK em AWGN

5.4 Taxa de erro de bit total, na borda e no centro de um sistema OFDM $/$ BPSK com OBO $=4 \mathrm{~dB}(\mathrm{IBO}=6.5 \mathrm{~dB})$ e $f_{d} T / N=0.003 . \quad 71$

5.5 Taxa de erro de bit total, na borda e no centro de um sistema OFDM $/$ QPSK com OBO $=4 \mathrm{~dB}(\mathrm{IBO}=6.5 \mathrm{~dB})$ e $f_{d} T / N=0.003 . \quad 72$

5.6 Taxa de erro de bit de um sistema OFDM/BPSK com OBO $=4 \mathrm{~dB}$ $(\mathrm{IBO}=6.5 \mathrm{~dB})$ e diferentes valores de $f_{d} T / N$.

5.7 Taxa de erro de bit de um sistema OFDM/QPSK com OBO $=4 \mathrm{~dB}$ e diferentes valores de $f_{d} T / N$. 
5.8 Taxa de erro de bit de um sistema OFDM/BPSK com $f_{d} T / N=$ 0.003 e diferentes valores de OBO.

5.9 Taxa de erro de bit de um sistema OFDM/QPSK com $f_{d} T / N=$ 0.003 e diferentes valores de OBO.

A.1 Modelo de não-linearidade com memória variante no tempo 